\title{
Water and acetaldehyde in HH212: The first hot corino in Orion
}

\author{
C. Codella ${ }^{1,2,3}$, C. Ceccarelli2,3,1, S. Cabrit ${ }^{4,2,3}$, F. Gueth ${ }^{5}$, L. Podio ${ }^{1}$, R. Bachiller ${ }^{6}$, F. Fontani ${ }^{1}$, \\ A. Gusdorf ${ }^{4}$, B. Lefloch ${ }^{2,3}$, S. Leurini ${ }^{7}$, and M. Tafalla ${ }^{6}$ \\ 1 INAF, Osservatorio Astrofisico di Arcetri, Largo E. Fermi 5, 50125 Firenze, Italy \\ e-mail: codella@arcetri.astro.it \\ 2 Univ. Grenoble Alpes, Institut de Planétologie et d'Astrophysique de Grenoble (IPAG), 38401 Grenoble, France \\ ${ }^{3}$ CNRS, Institut de Planétologie et d'Astrophysique de Grenoble (IPAG), 38401 Grenoble, France \\ ${ }^{4}$ LERMA, UMR 8112 du CNRS, Observatoire de Paris, École Normale Supérieure, 61 Av. de l'Observatoire, 75014 Paris, France \\ 5 IRAM, 300 rue de la Piscine, 38406 Saint Martin d'Hères, France \\ ${ }^{6}$ IGN, Observatorio Astronómico Nacional, Alfonso XII 3, 28014 Madrid, Spain \\ 7 Max-Planck-Institut für Radioastronomie, Auf dem Hügel 69, 53121 Bonn, Germany \\ Received 22 September 2015 / Accepted 18 December 2015
}

\section{ABSTRACT}

\begin{abstract}
Aims. Using the unprecedented combination of high resolution and sensitivity offered by ALMA, we aim to investigate whether and how hot corinos, circumstellar disks, and ejected gas are related in young solar-mass protostars.

Methods. We observed $\mathrm{CH}_{3} \mathrm{CHO}$ and deuterated water (HDO) high-excitation ( $E_{\mathrm{u}}$ up to $335 \mathrm{~K}$ ) lines towards the Sun-like protostar HH212-MM1.

Results. For the first time, we have obtained images of $\mathrm{CH}_{3} \mathrm{CHO}$ and $\mathrm{HDO}$ emission in the inner $\simeq 100$ AU of $\mathrm{HH} 212$. The multifrequency line analysis allows us to contrain the density $\left(\geq 10^{7} \mathrm{~cm}^{-3}\right)$, temperature $(\simeq 100 \mathrm{~K})$, and $\mathrm{CH}_{3} \mathrm{CHO}$ abundance $\left(\simeq 0.2-2 \times 10^{-9}\right)$ of the emitting region. The HDO profile is asymmetric at low velocities $\left(\leq 2 \mathrm{~km} \mathrm{~s}^{-1}\right.$ from $\left.V_{\text {sys }}\right)$. If the HDO line is optically thick, this points to an extremely small ( $\sim 20-40 \mathrm{AU})$ and dense $\left(\geq 10^{9} \mathrm{~cm}^{-3}\right)$ emitting region.

Conclusions. We report the first detection of a hot corino in Orion. The HDO asymmetric profile indicates a contribution of outflowing gas from the compact central region, possibly associated with a dense disk wind.
\end{abstract}

Key words. stars: formation - ISM: jets and outflows - ISM: molecules - ISM: individual objects

\section{Introduction: The HH212 laboratory}

The birth of a Sun-like star is a complex game played by several participants whose respective roles are not yet entirely clear. On the one hand, the star-to-be accretes matter from a collapsing envelope. It is commonly believed that the gravitational energy released in the process heats up the material surrounding the protostar, creating warm regions $(\sim 100 \mathrm{~K})$ enriched by complex organic molecules (COMs) called hot corinos (e.g. Ceccarelli et al. 2007). On the other hand, the presence of angular momentum and magnetic fields leads to two consequences: (i) the formation of circumstellar disks, also called protoplanetary disks; and (ii) substantial episodes of matter ejection (e.g. Frank et al. 2014, and references therein).

Despite the progress achieved in the last decade, to our knowledge only three hot corinos have been imaged so far in COMs on $\leq 100$ AU scale (IRAS 16293-2422, NGC 1333IRAS2A, and IRAS4A; e.g. Jørgensen et al. 2012; Maury et al. 2014; Taquet et al. 2015, and references therein). Hence, several questions about these three components (hot corino, circumstellar disk, and ejected material) are still unanswered. What is the origin and composition of the hot corinos: are they thermally or shocked heated regions? What is the origin of the ejections: are they due to disk or stellar winds? How are these three phenomena linked? In addition to the physical and evolutionary connection, the three phenomena have one thing in common: they all need to be studied using mm observations at high spatial resolution (on scales $\leq 100 \mathrm{AU}$ ).

$\mathrm{HH} 212$ is located in Orion (at $450 \mathrm{pc}$ ) and is one of the best laboratories in which to study the (Class 0) protostellar stage. The HH212-MM1 protostar is hidden in a rotating and infalling envelope (Wiseman et al. 2001; Lee et al. 2014) and drives a spectacular jet and outflow. It has been extensively studied with the SMA and IRAM PdBI on scales down to $\simeq 0 . ' 3-0$. .'4 (Lee et al. 2006, 2007, 2008; Codella et al. 2007; Cabrit et al. 2007,2012 ), showing a microjet with inner peaks at $\pm 1-2^{\prime \prime}=$ 450-900 AU of the protostar. Further observations performed with ALMA Early Science in Band 7 in $\mathrm{HCO}^{+}, \mathrm{C}^{17} \mathrm{O}$, and SO indicate a small-scale velocity gradient along the equatorial plane consistent with a rotating disk of $\simeq 0.2=90 \mathrm{AU}$ around a $\simeq 0.3 \pm 0.1 M_{\odot}$ source (Lee et al. 2014; Codella et al. 2014; Podio et al. 2015). The HH212 region is thus, so far, the only protostar where both a bright bipolar jet and a compact rotating disk have been revealed. Given its association with all the ingredients of the Sun-like star formation recipe, HH212 stands out as being the perfect target for investigating the link between hot corino, disk, and outflow in the protostellar stage.

In this Letter, we further exploit the ALMA dataset of Codella et al. (2014) to probe the inner region of $\mathrm{HH} 212$. Specifically, we report the detection of twelve high-lying $\left(E_{\mathrm{u}} \geq\right.$ $150 \mathrm{~K})$ lines from acetaldehyde $\left(\mathrm{CH}_{3} \mathrm{CHO}\right)$ and one $\left(E_{\mathrm{u}}=\right.$ $335 \mathrm{~K}$ ) line from deuterated water (HDO). They reveal the presence of a hot corino previously unknown in this source and, possibly, probe the base of a slow disk wind.

\section{Observations}

HH212 was observed at $850 \mu$ m with ALMA using $2412 \mathrm{~m}$ antennas on 2012 December 1 (Early Science Cycle 0 phase; Codella et al. 2014). The baselines were between $20 \mathrm{~m}$ and 


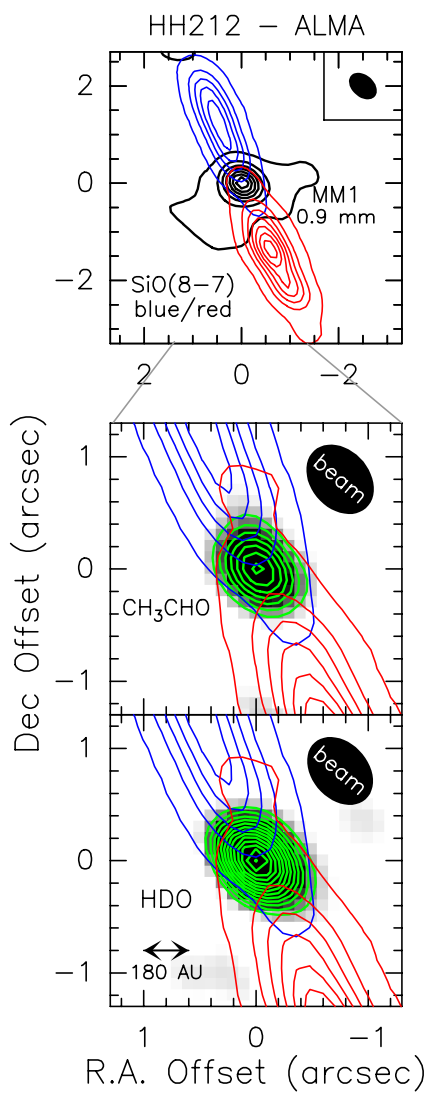

Fig. 1. Upper panel: the HH212 protostellar system as observed by ALMA-Band 7 (Codella et al. 2014). Blue/red contours plot the blue-/ redshifted $\mathrm{SiO}(8-7)$ jet at $\pm 8 \mathrm{~km} \mathrm{~s}^{-1}$ from $V_{\text {sys }}$, overlaid on the $0.9 \mathrm{~mm}$ continuum (black contours). Positions are with respect to the coordinates reported in Sect. 2. The filled ellipse shows the synthesised beam $(\mathrm{HPBW})$ for SiO: 0.63 $\times 00^{\prime} 46\left(\mathrm{PA}=49^{\circ}\right)$. Middle panel: zoom-in of the central region: the $\mathrm{CH}_{3} \mathrm{CHO}\left(18_{0,18}-17_{0,17}\right) \mathrm{A}$ emission integrated over $\pm 5 \mathrm{~km} \mathrm{~s}^{-1}$ (green contours and grey scale) overlaid on the $\mathrm{SiO}$ jet. First contours and steps are $5 \sigma\left(50 \mathrm{mJy}^{\mathrm{beam}}{ }^{-1} \mathrm{~km} \mathrm{~s}^{-1}\right)$ and $2 \sigma$, respectively. The HPBW is $0{ }^{\prime} 69 \times 00^{\prime} 52\left(\mathrm{PA}=42^{\circ}\right)$. Bottom panel: same as middle panel for HDO. The HPBW is $0{ }^{\prime} 68 \times 00^{\prime} 51\left(\mathrm{PA}=42^{\circ}\right)$.

$360 \mathrm{~m}$ with a maximum unfiltered scale of $3^{\prime \prime}$. The spectral windows $333.7-337.4 \mathrm{GHz}$ and $345.6-349.3 \mathrm{GHz}$ were observed using spectral units of $488 \mathrm{kHz}\left(0.42-0.44 \mathrm{~km} \mathrm{~s}^{-1}\right)$. Calibration was carried out following standard procedures, using quasars J0538-440, J0607-085, Callisto, and Ganymede. Spectral line imaging was achieved with the CASA package, while data analysis was performed using the GILDAS ${ }^{1}$ package. The continuum-subtracted images have a typical clean-beam FWHM of $0{ }^{\prime} 65 \times 00^{\prime} 47\left(\mathrm{PA}=35^{\circ}\right)$, and an rms noise level of 3-4 mJy beam ${ }^{-1}$ in $0.44 \mathrm{~km} \mathrm{~s}^{-1}$ channels. Positions are given with respect to the MM1 protostar continuum peak located at $\alpha(\mathrm{J} 2000)=05^{\mathrm{h}} 43^{\mathrm{m}} 51^{\mathrm{s}} .41, \delta(\mathrm{J} 2000)=-01^{\circ} 02^{\prime} 53^{\prime \prime} \cdot 17$ (Lee et al 2014).

\section{Results and discussion}

The ALMA $8 \mathrm{GHz}$ bandwidth presents a very rich spectrum with many lines in emission towards the MM1 protostar (Fig. 1) that have revealed a number of high-excitation molecular lines (see also Table 1), among which (i) twelve lines of acetaldehyde $\left(\mathrm{CH}_{3} \mathrm{CHO}\right)$ with $E_{\mathrm{u}}$ between $150 \mathrm{~K}$ and $200 \mathrm{~K}$ and (ii) the $3_{3,1}-4_{2,2}$ line of deuterated water (HDO) from $E_{\mathrm{u}}=335 \mathrm{~K}$

\footnotetext{
1 http://wWw.iram.fr/IRAMFR/GILDAS
}

Table 1. List of unblended transitions detected towards HH212-MM1 and used for the $\mathrm{CH}_{3} \mathrm{CHO}$ (LTE) and HDO (LVG) analysis.

\begin{tabular}{cccccc}
\hline \hline $\begin{array}{c}\text { Transition } \\
\left(J_{\mathrm{Ka}, \mathrm{Kc}}\right)\end{array}$ & $\begin{array}{c}v^{a} \\
(\mathrm{MHz})\end{array}$ & $\begin{array}{c}E_{\mathrm{u}}{ }^{a} \\
(\mathrm{~K})\end{array}$ & $\begin{array}{c}S \mu^{2 a} \\
\left(\mathrm{D}^{2}\right)\end{array}$ & $\begin{array}{c}\mathrm{rms}^{b} \\
(\mathrm{mK})\end{array}$ & $\begin{array}{c}F_{\mathrm{int}}{ }^{b} \\
\left(\mathrm{~K} \mathrm{~km} \mathrm{~s}^{-1}\right)\end{array}$ \\
\hline $18_{1,18}-17_{1,17} \mathrm{E}$ & 333941.4 & 155 & 226.8 & 51 & $2.8(0.5)$ \\
$17_{2,15}-16_{2,14} \mathrm{~A}$ & 334931.4 & 153 & 212.1 & 57 & $2.6(0.1)$ \\
$18_{0,18}-17_{0,17} \mathrm{~A}$ & 335358.7 & 154 & 226.8 & 52 & $2.3(0.5)$ \\
$18_{3,16}-17_{3,15} \mathrm{~A}$ & 347519.2 & 179 & 221.3 & 68 & $2.4(0.2)$ \\
$18_{3,16}-17_{3,15} \mathrm{E}$ & 347563.3 & 179 & 221.1 & 68 & $1.7(0.3)$ \\
\hline \multicolumn{7}{c}{$\mathrm{HDO}$} \\
\hline $3_{3,1}-4_{2,2}$ & 335395.5 & 335 & 0.4 & 57 & $4.9(0.2)$ \\
\hline
\end{tabular}

Notes. ${ }^{(a)}$ From the JPL database (Pickett et al. 1998). ${ }^{(b)}$ In $T_{\mathrm{B}}$ scale.

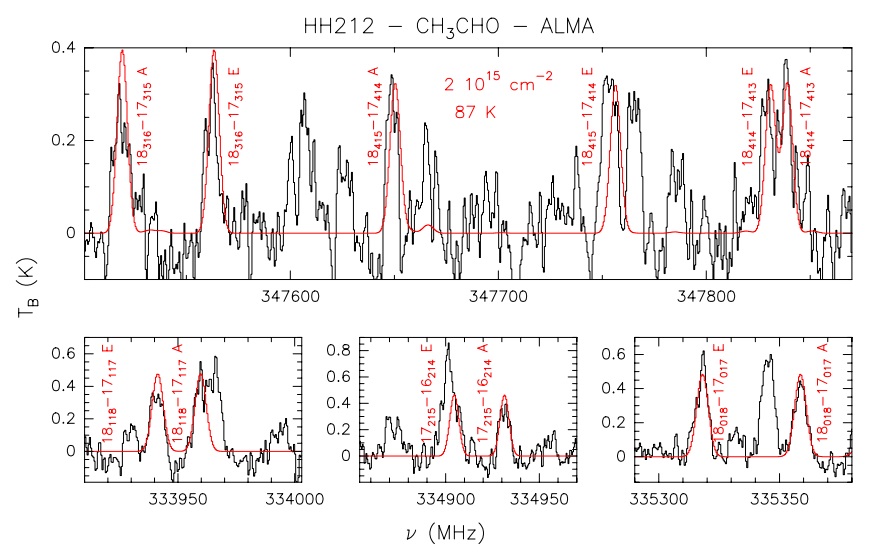

Fig. 2. $\mathrm{CH}_{3} \mathrm{CHO}$ emission (in $T_{\mathrm{B}}$ scale) extracted at the MM1 position. The four panels show the frequency intervals where the $\mathrm{CH}_{3} \mathrm{CHO}$ lines are located (see Table 1 for those unblended with other lines). The red line shows the synthetic spectrum which best reproduces the observations assuming LTE and optically thin emission obtained with the GILDAS-Weeds package (Maret et al. 2011) with source size $=00^{\prime} 3$, $T_{\text {rot }}=87 \mathrm{~K}, N_{\mathrm{CH}_{3} \mathrm{CHO}}=2 \times 10^{15} \mathrm{~cm}^{-2}, \mathrm{FWHM}$ linewidth $=5.0 \mathrm{~km} \mathrm{~s}^{-1}$, and LSR velocity $=+1.7 \mathrm{~km} \mathrm{~s}^{-1}$ (see footnote 2 ).

(see Figs. 2 and 3). The lines were identified using the Jet Propulsion Laboratory (JPL) molecular database (Pickett et al. 1998). Figure 1 shows that $\mathrm{CH}_{3} \mathrm{CHO}$ and HDO are only observed towards the protostellar position and are spatially unresolved. For the first time these high-excitation molecular lines have been detected towards the $\mathrm{HH} 212$ inner region revealing the existence of hot gas around the MM1 protostar driving the bipolar $\mathrm{SiO}$ jet. The line profiles peak in the $+1.2,+2.0 \mathrm{~km} \mathrm{~s}^{-1}$ range, i.e. close to the systemic velocity ${ }^{2} V_{\text {sys }}$, which is also quite broad $\left(F W H M \simeq 5-6 \mathrm{~km} \mathrm{~s}^{-1}\right)$. We now examine the constraints brought by $\mathrm{CH}_{3} \mathrm{CHO}$ and $\mathrm{HDO}$ on the nature and physi$\mathrm{cal} / \mathrm{chemical}$ conditions of the emission region(s).

\section{1. $\mathrm{CH}_{3} \mathrm{CHO}$ emission}

The $\mathrm{CH}_{3} \mathrm{CHO}$ emission is symmetric around the systemic velocity and can be explained by assuming that it originates in the hot corino, namely the region where the dust temperature is high

2 In the literature, values between +1.3 and $+1.8 \mathrm{~km} \mathrm{~s}^{-1}$ are reported for the $V_{\text {sys }}$ of $\mathrm{HH} 212$. The value of $+1.3 \mathrm{~km} \mathrm{~s}^{-1}$ adopted in Codella et al. (2014) was affected by a shift in the frequency scale; we adopt here $+1.7 \mathrm{~km} \mathrm{~s}^{-1}$ (Lee et al. 2014). However, the results are not all dependent on this choice. 


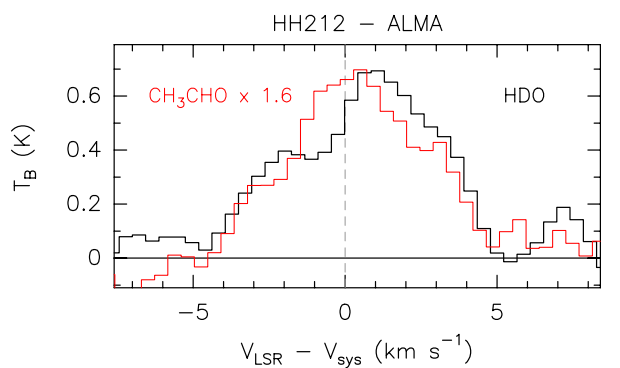

Fig. 3. Comparison between $\operatorname{HDO}\left(3_{3,1}-4_{2,2}\right)$ (black line) and $\mathrm{CH}_{3} \mathrm{CHO}\left(18_{0,18}-17_{0,17}\right) \mathrm{A}$ (red, multiplied by a factor of 1.6) as observed towards HH212-MM1 (in $T_{\mathrm{B}}$, scale). The vertical dashed line defines the velocity with respect to $V_{\text {sys }}=+1.7 \mathrm{~km} \mathrm{~s}^{-1}$ (see footnote 2).

enough $(\geq 100 \mathrm{~K})$ to sublimate the frozen ice mantles. Given that the $\mathrm{CH}_{3} \mathrm{CHO}$ emission is spatially unresolved, we assume a source size equal to half a beam, namely 0.33 . A local thermodynamic equilibrium (LTE) optically thin analysis of $\mathrm{CH}_{3} \mathrm{CHO}$ (using only lines with less than $30 \%$ contamination from blended lines, in terms of integrated emission, see Table 1) indicates temperature of $87 \pm 47 \mathrm{~K}$ and a column density of $2 \pm 1 \times 10^{15} \mathrm{~cm}^{-2}$ (see the the rotational diagram in Fig. A.1 and the synthetic spectra in Fig. 2). With these values the opacity is less than 0.4. In the case of a smaller emitting size, e.g. 0.'06 (as assumed for HDO in Sect. 3), $N_{\mathrm{CH}_{3} \mathrm{CHO}}$ increases by a factor of $\sim 20$.

Unfortunately, we cannot use the continuum emission to estimate the $\mathrm{H}_{2}$ column density (and hence the $\mathrm{CH}_{3} \mathrm{CHO}$ abundance) because the submillimetre continuum peak seen in interferometric maps is optically thick (Codella et al. 2007; Lee et al. 2008, 2014). Taquet et al. (2015) measured $\mathrm{N}\left(\mathrm{H}_{2}\right) \simeq$ $10^{24}-10^{25} \mathrm{~cm}^{-2}$ towards the NGC 1333-IRAS2A and IRAS4A hot corinos on a spatial scale of $\sim 470 \mathrm{AU}$, i.e. a factor $\sim 3$ higher than is sampled here. If we take these values and 0.3 as emitting size, then $X_{\mathrm{CH}_{3} \mathrm{CHO}} \sim 0.2-2 \times 10^{-9}$, in agreement with the value recently derived in the IRAS $16293-2422$ hot corino (using single-dish data; $3 \times 10^{-9}$ : Jaber et al. 2014).

\subsection{HDO emission}

Figure 3 compares the HDO profile derived towards MM1 with that of $\mathrm{CH}_{3} \mathrm{CHO}\left(18_{0,18}-17_{0,17}\right) \mathrm{A}$; we chose this transition as representative of the acetaldehyde lines suffering no contamination. At low velocity $\left(\leq 2 \mathrm{~km} \mathrm{~s}^{-1}\right.$ from $\left.V_{\text {sys }}\right)$ the HDO profile shows an asymmetry; the redshifted emission is clearly brighter than the blueshifted. The line profile is determined by the kinematics and, in case of high optical thickness, by radiative effects such as self-absorption. In order to understand the relative importance of each, we study two limit situations: (i) the optically thin case where the kinematics will dominate and (ii) the optically thick case where the profile will be strongly affected by radiative effects. To this end, we compare the observed emission with that predicted by a non-LTE large velocity gradient (LVG) model using the code by Ceccarelli et al. (2003), a plane parallel geometry, the collisional coefficients for the system $\mathrm{HDO}-\mathrm{H}_{2}$ computed by Faure et al. $(2012)^{3}$, and assuming a Boltzmann distribution for the ortho-to-para $\mathrm{H}_{2}$ ratio $^{4}$. Figure 4 shows the

\footnotetext{
3 The collisional coefficients are extracted from the BASECOL database, Dubernet et al. (2013).

4 The collisional coefficients with ortho- $\mathrm{H} 2$ are a factor of 5 larger than the corresponding rate coefficients with para-H2 (Faure et al. 2012) at low temperatures ( $\ll 45 \mathrm{~K}$ ) (Faure et al. 2012), while they are similar at higher temperatures.
}

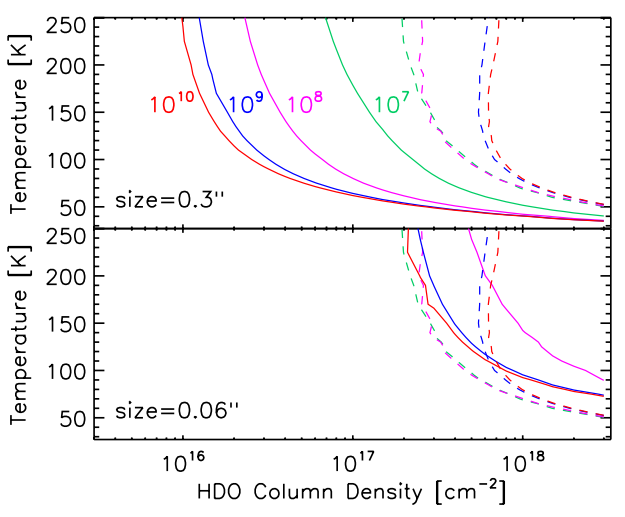

Fig. 4. LVG predictions of the temperature versus HDO column density required to reproduce the observed velocity-integrated emission (solid curves) and to have unit line opacity (dashed curves) for densities of: $10^{7} \mathrm{~cm}^{-3}$ (green), $10^{8} \mathrm{~cm}^{-3}$ (magenta), $10^{9} \mathrm{~cm}^{-3}$ (blue), and $10^{10} \mathrm{~cm}^{-3}$ (red). Optical depth increases with column density. The upper panel refers to a source size of 0.3 and the lower panel to $0{ }^{\prime} 06$.

temperature versus the HDO column density predicted to generate the observed s signal (see Table 1). Given that the HDO emission is spatially unresolved, the upper panel shows the case of a source size equal to half a beam $\left(0^{\prime} .^{\prime} 3\right)$ and the lower panel shows a case with a size that is lower by a factor of five (see Sect. 3.2.2). The plots cover densities from $10^{7}$ to $10^{10} \mathrm{~cm}^{-3}$. The highdensity end, $10^{10} \mathrm{~cm}^{-3}$, represents LTE conditions. The low end, $10^{7} \mathrm{~cm}^{-3}$, is set by the constraint that the HDO abundance has to be smaller than $10 \%$ of the $\mathrm{D} / \mathrm{H}$ elemental abundance (in agreement with previous observations of $\mathrm{HDO} / \mathrm{H}_{2} \mathrm{O}$ towards hot corinos, assuming an initial $\mathrm{H}_{2} \mathrm{O}$ abundance of $3 \times 10^{-5}$; see Taquet et al. 2014, 2015), namely $N(\mathrm{HDO}) / N\left(\mathrm{H}_{2}\right) \leq 3.2 \times 10^{-6}$, where $N\left(\mathrm{H}_{2}\right)$ is consistently derived from the density and size of the source. For a given size, this provides a limit to the permitted density- $N(\mathrm{HDO})$ values. In particular, for a size of 0.3 , if the density is $10^{6} \mathrm{~cm}^{-3}$, then $N\left(\right.$ HDO) should be $\leq 7 \times 10^{15} \mathrm{~cm}^{-2}$. We find that it is not possible, then, to reproduce the HDO line intensity and we rule out a density of $10^{6} \mathrm{~cm}^{-3}$. On the other hand, at $10^{7} \mathrm{~cm}^{-3}, N(\mathrm{HDO})$ has to be $\leq 7 \times 10^{16} \mathrm{~cm}^{-2}$, and the temperature has to be larger than $230 \mathrm{~K}$ (see Fig. 4). Armed with these predictions, we now discuss the implications of each assumption, the optically thin and thick HDO line.

\subsubsection{Optically thin case}

If the line is optically thin, then the observed HDO asymmetry at low velocities must be caused by intrinsic brightness asymmetries between the red- and blueshifted components in the beam. We examine various explanations in turn.

First, the HDO emission might probe the rotating disk, whose density is expected to be larger than $\sim 10^{7} \mathrm{~cm}^{-3}$ (Lee et al. 2014) and whose $\mathrm{C}^{17} \mathrm{O}$ and $\mathrm{SO}$ emissions also extend to $\pm 5 \mathrm{~km} \mathrm{~s}^{-1}$ (Codella et al. 2014; Podio et al. 2015). However, the low-velocity range of the HDO line being due to the protostellar disk is not supported by the large (factor of 2) difference between (i) the blue and red peak brightnesses and (ii) the blue and red peak velocities $\left(V_{\mathrm{LSR}}-V_{\text {sys }}\right.$ at $\leq+1$ and $\left.\sim-2 \mathrm{~km} \mathrm{~s}^{-1}\right)$. This would require a high degree of non-axisymmetry in the molecular disk emission, which has never been seen at this level in the profiles of younger disks, not even when tidally disturbed (see e.g. the RW Aur disk profile in Fig. 4 of Cabrit et al. 2006), although it has been revealed in some evolved transitional disks with prominent dust traps (e.g. Casassus et al. 2013). 
We can also rule out an origin from the rotating infalling envelope; infall motions of $1 \mathrm{~km} \mathrm{~s}^{-1}$ arise from radii 0 .' 3 (Lee et al. 2014), which would lead to spatially resolved peak emission, unlike the observed emission. In addition, in this case, there is no reason for the redshifted emission being definitely brighter than the blue.

The last and most likely possibility is that the lowvelocity HDO emission is dominated by outflowing motions. The $\mathrm{SiO}(8-7)$ profile towards MM1 derived from the same ALMA dataset (Podio et al. 2015) is definitely too broad (up to $\pm 20 \mathrm{~km} \mathrm{~s}^{-1}$ ) for HDO to probe the inner portion of the fast jet traced by $\mathrm{SiO}$ (Fig. 1). On the other hand, channel maps of SO $10_{11}-10_{10}\left(E_{\mathrm{u}}=143 \mathrm{~K}\right)$ have clearly revealed a low-velocity compact bipolar outflow (Podio et al. 2015) in the $\pm 2 \mathrm{~km} \mathrm{~s}^{-1}$ ( $\leq 25 \mathrm{~km} \mathrm{~s}^{-1}$ after deprojection) range. Hence, it seems quite plausible that the low-velocity HDO would trace the inner region $(\leq 0,3$, i.e. $138 \mathrm{AU})$ of a more extended outflow heated by the protostar at a temperature high enough (at least $100 \mathrm{~K}$ ) to release icy dust mantles and with a brighter HDO line in its red lobe.

\subsubsection{Optically thick case}

If we assume that the HDO line shape is determined by the line opacity rather than pure kinematics, then the line asymmetry is due to blueshifted absorption, revealing again outflowing gas (because an optically thick infalling envelope would lead to redshifted absorption). We can obtain stringent constraints on the size, density, temperature, and HDO abundance of the emitting gas. To this end, in the non-LTE LVG predictions in Fig. 4 we add dashed curves where the HDO line opacity is equal to unity, i.e. we assume a moderate thickness to explain the line absorption. Also, the lower level of the HDO transition is very high $\left(E_{1}=318 \mathrm{~K}\right)$ : the upper panel of Fig. 4 shows that if the source size is 0.3 , the $\tau=1$ curves (dashed) are always shifted to the right of the curves of the observed signal (solid) and never overlap: in other words, any optically thick layer of this size (or larger) would emit more in HDO than observed in our beam. In order to avoid excessive emission, i.e. to have the two families of curves intersect, and to constrain that $N(\mathrm{HDO}) / N\left(\mathrm{H}_{2}\right)$ does not exceed the $10 \%$ of the $\mathrm{D} / \mathrm{H}$ elemental abundance, one needs to assume a larger beam dilution factor requiring a source size smaller than 0.'08 (37 AU) and larger than 0.'04 (18 AU). The lower panel of Fig. 4 shows the intermediate case, with 0 .'06 (28 AU). The solution is, in this case, for temperatures between $70 \mathrm{~K}$ and $110 \mathrm{~K}$ and densities of $10^{9}-10^{10} \mathrm{~cm}^{-3}$. In particular, for a temperature around $100 \mathrm{~K}, N(\mathrm{HDO}) \sim 7 \times 10^{17} \mathrm{~cm}^{-2}$, corresponding to $N(\mathrm{HDO}) / N\left(\mathrm{H}_{2}\right)=1.7 \times 10^{-6}-10^{-7}$. Assuming a typical water abundance of $\sim 10^{-4}$ would imply $\mathrm{HDO} / \mathrm{H}_{2} \mathrm{O} \sim$ $1.7 \times 10^{-2}-10^{-3}$, consistent with the values measured in hot corinos (Persson et al. 2014; Ceccarelli et al. 2015). The optically thick case thus also requires slow outflowing gas, further constraining the physical conditions, i.e. a size $\simeq 18-37 \mathrm{AU}$, $T_{\text {kin }} \simeq 100 \mathrm{~K}$, and $n_{\mathrm{H}_{2}} \geq 10^{9} \mathrm{~cm}^{-3}$.

\section{Conclusions}

The combination of high sensitivity, high angular resolution, and large bandwidth offered by ALMA has allowed us to image high-excitation ( $E_{\mathrm{u}}$ up to $335 \mathrm{~K}$ ) $\mathrm{CH}_{3} \mathrm{CHO}$ and $\mathrm{HDO}$ emission in the inner $\simeq 100 \mathrm{AU}$ of the Sun-like $\mathrm{HH} 212$ protostar for the first time. Both $\mathrm{HDO}$ and $\mathrm{CH}_{3} \mathrm{CHO}$ emission indicates $T_{\text {kin }}$ larger than $70 \mathrm{~K}$, while HDO requires gas densities $\geq 10^{7} \mathrm{~cm}^{-3}$. We thus report the detection of the first hot corino in Orion. The acetaldehyde abundance is similar to that measured in hot corinos located in low-mass star forming regions in Ophiuchus.

The asymmetric HDO profile at low velocities indicates that at least some of the deuterated water is associated with slow outflowing gas, where a high SO abundance (up to $10^{-7}$ ) has also been detected by Podio et al. (2015). These finding support a chemical enrichment of the low-velocity outflowing gas heated by the protostar in its surroundings. If the emission is optically thick the emitting size must be very small (18-37 AU) and the density has to be extremely high, with $n_{\mathrm{H}_{2}} \geq 10^{9} \mathrm{~cm}^{-3}$. With such extreme sizes and densities, it is tempting to speculate that the observed gas may be associated with a disk wind gas accelerated at the base. Interestingly, the occurrence of a wideangle flow in $\mathrm{HH} 212$ with a nested onion-like velocity structure has recently been suggested by $\mathrm{C}^{34} \mathrm{~S}$ observations with ALMA (Codella et al. 2014).

Acknowledgements. We acknowledge the anonymous referee for instructive comments. This paper makes use of the ADS/JAO.ALMA\#2011.0.000647.S data (PI: C. Codella). ALMA is a partnership of ESO (representing its member states), NSF (USA), and NINS (Japan), together with NRC (Canada) and NSC and ASIAA (Taiwan), in cooperation with the Republic of Chile. The Joint ALMA Observatory is operated by ESO, AUI/NRAO, and NAOJ. This work was partly supported by the PRIN INAF 2012 - JEDI and by the Italian Ministero dell'Istruzione, Università e Ricerca through the grant Progetti Premiali 2012 - iALMA. L.P. has received funding from the European Union Seventh Framework Programme (FP7/2007-2013) under grant agreement No. 267251.

\section{References}

Cabrit, S., Pety, J., Pesenti, N., \& Dougados, C. 2006, A\&A, 452, 897

Cabrit, S., Codella, C., Gueth, F., et al. 2007, A\&A, 468, L29

Cabrit, S., Codella, C., Gueth, F., \& Gusdorf, A. 2012, A\&A, 548, L2

Casassus, S., van der Plas, G., M. Perez, S., et al. 2013, Nature, 493, 191

Ceccarelli, C., Maret, S., Tielens, A. G. G. M., Castets, A., \& Caux, E. 2003, A\&A, 410, 587

Ceccarelli, C., Caselli, P., Herbst, E., Tielens, A. G. G. M., \& Caux, E. 2007, Protostars and Planets V (Tucson: University of Arizona), 47

Ceccarelli, C., Caselli, P., Bockeliée-Morvan, D., et al. 2015, Protostars and Planets VI (Tucson: University of Arizona), 859

Codella, C., Cabrit, S., Gueth, F., et al. 2007, A\&A, 462, L53

Codella, C., Cabrit, S., Gueth, F., et al. 2014, A\&A, 568, L5

Dubernet, M.-L., Alexander, M. H., Ba, Y. A., et al. 2013, A\&A, 553, 50

Faure, A., Wiesenfeld, L., Scribano, Y., \& Ceccarelli, C. 2012, MNRAS, 420, 699

Frank, A., Ray, T. P., Cabrit, S., et al. 2014, Protostars and Planets VI (Tucson: University of Arizona), 451

Jaber, A. A., Ceccarelli, C., Kahane, C., \& Caux, E. 2014, ApJ, 791, 29

Jørgensen, J. K., Favre, C., Bisschop, S. E., et al. 2012, ApJ, 757, L4

Lee, C.-F., Ho, P. T. P., Beuther, H., et al. 2006, ApJ, 639, L292

Lee, C.-F., Ho, P. T. P., Hirano, N., et al. 2007, ApJ, 659, L499

Lee, C.-F., Ho, P. T. P., Bourke, T. L., et al. 2008, ApJ, 685, 1026

Lee, C.-F., Hirano, N., Zhang, Q., et al. 2014, ApJ, 786, 114

Maret, S., Hily-Blant, P., Pety, J., Bardeau, S., \& Raynier, E. 2011, A\&A, 526, A47

Maury, A. J., Belloche, A., André, Ph., et al. 2014, A\&A, 563, L2

Persson, M. V., Jørgensen, J. K., van Dishoeck, E. F., \& Harsono, D. 2014, A\&A, 563, A74

Pickett, H. M., Poynter, R. L., Cohen, E. A., et al. 1998, J. Quant. Spectr. Rad. Transf., 60, 883

Podio, L., Codella, C., Gueth, F., et al. 2015, A\&A, 581, 85

Taquet, V., Charnley, S. B., Steven, B., \& Sipilä, O. 2014, ApJ, 791, 1

Taquet, V., López-Sepulcre, A., Ceccarelli, C., et al. 2015, ApJ, 804, 81

Wiseman J., Wootten, A., Zinnecker, H., \& McCaoughrean, M. 2001, ApJ, 550, L87 


\section{Appendix A: The $\mathrm{CH}_{3} \mathrm{CHO}$ rotational diagram}

Table 1 lists the emission lines observed towards HH212-MM1 and used for the standard analysis of the rotational diagram (Fig. A.1), which allows us to derive $T_{\text {rot }}=87 \pm 47 \mathrm{~K}$ and $N_{\text {tot }}=2 \pm 1 \times 10^{15} \mathrm{~cm}^{-2}$.

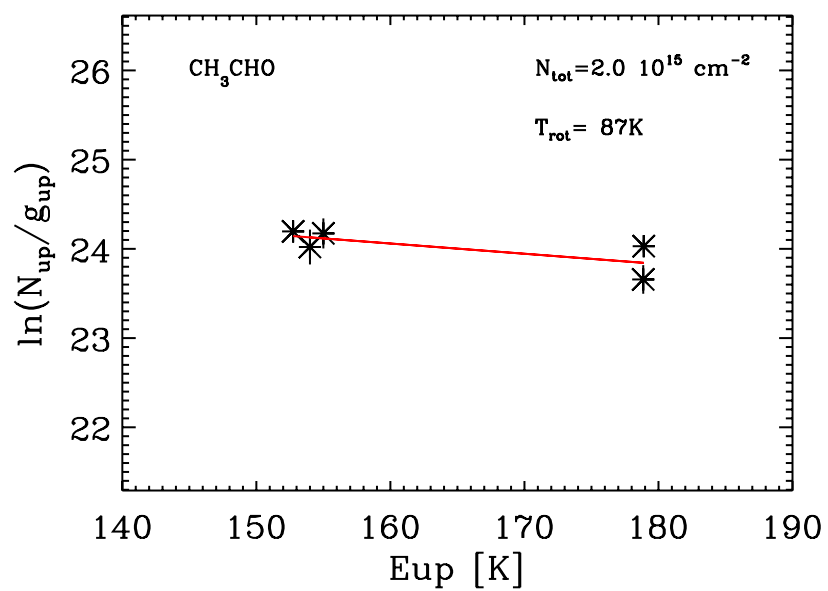

Fig. A.1. Rotation diagram for the $\mathrm{CH}_{3} \mathrm{CHO}$ transitions reported in Table 1 . The parameters $N_{\text {up }}, g_{\text {up }}$, and $E_{\text {up }}$ are respectively the column density, the degeneracy, and the energy of the upper level. We note that the error bars on $\ln \left(N_{\text {up }} / g_{\text {up }}\right)$ are given by the vertical bar of the symbols. The $g_{\text {up }}$ value is 74 for all the transitions used here (and reported in Table 1) with the exception of $17_{2,15}-16_{2,14} \mathrm{~A}$, where $g_{\text {up }}=70$. The plot allows us to derive a rotational temperature of $87 \pm 47 \mathrm{~K}$ and a total column density of $2 \pm 1 \times 10^{15} \mathrm{~cm}^{-2}$. 